Stanko Gerjolj

University of Ljubljana, Slovenia

\title{
Biblische Familien als Herausforderung für die Erziehung heute
}

\section{Biblical Families as a Challenge for Contemporary Education}

\begin{abstract}
The Bible tells us not only about God, but about human beings and anthropological relationships. By reflecting on relationships of some biblical families, we can gain the potentials of overcoming different kinds of deep relational conflicts. In this way we might learn how to approach Jeremiah's prophetic oracle: No longer will anyone go around saying, "Sour grapes eaten by parents leave a sour taste in the mouths of their children" (Jer. 31:29). According to the stories about Abraham and his family, Isaac and his family, Jacob and his family, and Josef and his reconciliation with his brothers in Egypt, we see that the "taste of the grapes" from the previous generations undoubtedly affects the next generation. To overcome the negative consequences and to prevail over the compulsive repetitions of the past generations, the biblical stories have lessons that cannot be ignored, lessons that are didactic even to contemporary men and women, especially in the context of the crisis which family life is experiencing today. By accepting the history and in learning from it, which includes at least some levels of forgiveness and reconciliation with the past, the next generation can potentiality cultivate the ability to "live different" and to "do better". In that context God is giving the chance for a "new life" to each new generation.
\end{abstract}

\section{Keywords}

Family, education, generation, conflict, reconciliation, integration.

Ganzheitlich orientierte Begegnungen mancher jüdischer, vor allem biblischer Geschichten und Erzählungen können zu Ressourcen leiten, die sowohl in pädagogisch-didaktischen als auch in beraterischen und therapeutischen Tätigkeiten unterstützend wirken und Lebenskräfte wecken, die zu persönlichen Wandlungsprozessen führen und für die Verarbeitung 
von Lebenskrisen hilfreich sind. Die Behauptung - die Bibel sei das Buch des Lebens - lehnt sich nicht an die mögliche Verbreitung des Buches, sondern in erster Linie an die Tatsache, dass der Mensch in diesem Buch Botschaften und Anregungen herauslesen kann, die ihm zu eigener Selbstfindung verhelfen. Unabhängig davon, ob wir der Bibel als Heils-Geschichte, als CredoGeschichte oder als Literatur-Geschichte begegnen, treffen wir gewaltige Dramen an, die uns als Einzelperson, Familienmitglied, partnerschaftliches Lebewesen, Vater, Mutter, Kind, gemeinschaftsstiftendes Lebewesen, Mitglied einer Nation, einer Kultur, einer Generation etc. ansprechen und nicht zuletzt erziehungspsychologisch zum Nachdenken, zum Reflektieren und meistens auch zum wandelnden Handeln bewegen.

Die biblischen Figuren laden zur Identifikation regelrecht ein ${ }^{1}$. Ungeachtet dessen, ob es sich dabei um konkrete und historische Personen oder um mythische Gestalten handelt, tragen sie als eine Art Archetypen in sich eine grundlegende Bedeutung und vermitteln universelle Botschaften. Die biblischen Figuren warten auf den Leser, um sich ihm mitzuteilen ${ }^{2}$. Durch sie wird er aus einer verträumten Realität geweckt und in einen Traum geführt, der die Realität verändert. In diesem Sinne sind die biblischen Geschichten auch dann real, wenn sie in der beschriebenen Form physisch nie stattgefunden haben ${ }^{3}$. Gemäß dem Verständnis des Persönlichkeitswachstums in der jüdisch-christlichen Tradition stellen wir nicht nur fest, dass alles wahr ist, was in der Bibel steht, sondern wir werden durch ein tiefgehendes und meditatives Lesen auch zur existenziellen Erkenntnis geführt, dass in der Heiligen Schrift „alle“ Wahrheit präsent ist".

Der religiöse Charakter der biblischen Sprache will gerade die Ansicht hervorheben, dass es Lebensumstände und -bestimmungen gibt, die einerseits sehr wohl mit den Lebenseinstellungen und -entscheidungen einzelner

1 Vgl. A. Höfer, Spuren Gottes in meinem Leben, München 2003, S. 23.

2 Vgl. W. G. Rollins, Soul and Psyche: The Bible in Psychological Perspective, Minneapolis 1999, S. 150.

3 Vgl. M. A. Corey, Job, Jonah, and the Unconscious: A Psychological Interpretation of Evil and Spiritual Growth in the Old Testament, Lanham - New York - London 1995, S. 6.

4 Diese existenzielle Wahrheit offenbart sich überzeugend, wenn wir imstande sind, das Lesen und Reflektieren einzelner Teile und Absätze in eine Beziehung zur gesamten biblischen Botschaft zu stellen und sie nicht aus dem Kontext zu reißen. Vgl. R. E. Friedman, Commentary on the Torah: With a new English Translation, San Francisco 2001, S. 3; Vgl. H. Cloud, J. Townsend, How People Grow: What the Bible Reveals about Personal Growth, Grand Rapids - Michigan 2001, S. 20. 
Menschen zusammenhängen, andererseits aber zugleich Generationen hinweg das Leben prägen, indem neue Generationen „Botschaften“ vermittelt bekommen, die sowohl lebensstiftend als auch lebenshindernd sein können. In diesem Zusammenhang spricht die Bibel vom Segen (lebensstiftende Botschaften) und von „Verdammung“ (lebenshindernde Botschaften) (Gen 12, 2; Gen 9, 25). Dabei ist jede neue Generation dazu berufen, in glaubender Kommunikation mit Gott die (erhaltenen) lebensstiftenden Botschaften und Kräfte zu pflegen und aufzubauen und die (ebenso erhaltenen) lebenshindernden Botschaften und Ängste durch Annehmen, daraus Lernen und aufgrund dessen durch „Anders-Tun“ zu überwinden. Ist die jeweilige neue Generation anhand der verarbeiteten Vergangenheit befähigt und motiviert, mit Gott den „neuen Bund“ abzuschließen, wird ,in jenen Tagen“ das von Jeremias zitierte Sprichwort ,die Väter haben saure Trauben gegessen und den Söhnen werden die Zähne stumpf“ (Jer 31, 29) nicht mehr gesagt bekommen. Dass hier die neutestamentliche Lebensphilosophie verankert ist, nach der die persönliche Verantwortung für das Leben bekräftigt wird, steht ohne Zweifel, denn ,nur dem, der die sauren Trauben isst, werden die Zähne stumpf" (Jer 31, 30).

So sehr die von Jeremias anstiftende Hoffnung auch ermutigend wirken mag, verbleiben wir im realen Leben trotzdem nach wie vor auch von der alttestamentlichen Logik geprägt. Die bei Jeremias angedeutete neutestamentliche Christuszentrierte Hoffnungsdynamik baut nicht auf einer tabuisierenden Ablehnung der (familiären) Vergangenheit, sondern vielmehr auf dem Annehmen und Verarbeiten der (Ur)Elternbotschaften auf (Mt 5, 17).

Ein beispielhafter Beziehungszyklus, der das Leben mehrerer Generationen prägt, dabei Konflikte schürt und löst, sind sicherlich die Generationen von Abraham bis Josef aus Ägypten. Diese beinhalten in vielen Hinsichten archetypische Rollen und Lebenszüge, die in den gegenwärtigen Generationen genauso zum Tragen kommen können wie in den alttestamentlichen biblischen Lebensverhältnissen.

\section{Erste Generation: Abra/ha/m und Sara/i}

Abram ist kein fehlerloser und vollkommener Mensch, wohl aber eine zuhörende und lernfähige Persönlichkeit. Die Berufung Gottes bedeutete für Abram die Herausforderung zum ganzheitlichen Lebenswandel und 
Neubeginn, was eine totale Trennung von seinem Land und Vaterhaus sowie von der Verwandtschaft, d. h. vom alten Lebensstil, veranlasste ${ }^{5}$. Dabei ist der Aufbruch wichtiger als die Ankunft ${ }^{6}$, was man als Ruf zum Identitätsveränderungsprozess ${ }^{7}$ und im Kontext mit Lebensvisionen der Jugendlichen als ,gehe in dich und werde selbständig“ verstehen kann". Allerdings schließt der Hinweis ,das Land wird ihm von Gott gezeigt“ seine Offenheit und Bereitschaft, die Beziehung zu Gott, die ohne sensible zwischenmenschliche Beziehungen nicht denkbar ist, feinfühlig zu pflegen, ein. Das versprochene „Land“ symbolisiert also das „Land“ der selbständigen und beglückenden, dauerhaften und fruchtbaren Beziehungen“, worauf sich Abram erwartungsgemäß ,gesegnet" fühlt".

Wie echt und fest Ehebeziehungen sind, stellt sich in Prüfungen heraus. Dies zeigte sich auch bei Abram und seiner Frau Sarai. Ihr Verhalten in der Not ist vielen heutigen Beispielen verblüffend ähnlich.

Wie gewöhnlich, stellte sich auch Abram seinen Neubeginn etwas leichter und idyllischer vor als seine nahe Zukunft dann tatsächlich verlief. Um den aushungernden Herausforderungen, die auch als mangelnde Liebe, Zuneigung und Achtung verstanden werden kann, auszuweichen und die Anfangskrise zu überbrücken, suchte er sich vorübergehend eine andere, zwar selbstbefremdende, jedoch verlockende Realität im fremden Ägypten aus ${ }^{10}$. Aus Angst um sein Leben - Hass und Konflikte können auch „erschlagen“ - verkaufte er seine Frau Sarai als seine Schwester an den Pharao ${ }^{11}$. Da allerdings die verlogene, bis zur Gänze von der damaligen

5 „Der Herr sprach zu Abram: Zieh weg aus deinem Land, von deiner Verwandtschaft und aus deinem Vaterhaus in das Land, das ich dir zeigen werde“. Gen 12,1.

6 Vgl. R. E. Friedman, Commentary on the Torah: With a new English Translation, San Francisco 2001, S. 48.

7 Vgl. J. F. D. Creach, Violence in Scripture, Louisville - Kentucky 2013, S. 45.

8 Vgl. N. J. Cohen, Voices from Genesis: Guiding Us Through the Stages of Life, Woodstock, Vermont 1998, S. 73.

9 „Ich werde dich zu einem großen Volk machen, dich segnen und deinen Namen groß machen". Gen 12, 2 a.

${ }^{10}$ „Als über das Land eine Hungersnot kam, zog Abram nach Ägypten hinab“. Gen 12, 10a.

11 „Als er sich Ägypten näherte, sagte er zu seiner Frau Sarai: Ich weiß, du bist eine schöne Frau. Wenn dich die Ägypter sehen, werden sie sagen: Das ist seine Frau!, und sie werden mich erschlagen, dich aber am Leben lassen. Sag doch, du seiest meine Schwester, damit es mir deinetwegen gut geht und ich um deinetwillen am Leben bleibe. ... Da holte man die Frau in den Palast des Pharao. Er behandelte Abram ihretwegen gut". Gen 12, 11-16a. 
„Marktwirtschaft" beeinflusste Gesellschaft nicht lange funktionieren konnte $^{12}$, bekam Abram seine Frau zurück und musste ins Land seiner Startposition zurückkehren ${ }^{13}$.

\subsection{Partnerschaft und Nachwuchs}

Der zweite Versuch, einen neuen Lebensweg einzuschlagen, war im Vergleich zum ersten bedeutend ernsthafterer und engagierter. Allerdings musste Abram zur Kenntnis nehmen, dass seine Frau Sarai unfruchtbar war ${ }^{14}$, worauf er auf Vorschlag Sarais ihre Magd Hagar zu sich nahm und mit ihr den Sohn Ismael hatte.

Interessanterweise bekam Sarai für ihren „Seitensprung“ das erste Signal bei Abram und Abram für seinen Schritt der Untreue bei Sarai, was darauf hinweist, dass für eine Ehebeziehung immer „beide Seiten“ verantwortlich $\operatorname{sind}^{15}$. Auf alle Fälle sind sich nun alle beide ihrer Schuld und Schwäche bewusst und in dieser „sündhaften“ Krise wagen sie wieder einen Neubeginn. So „erging das Wort des Herrn in einer Vision an Abram“ und versprach ihm einen Sohn und zahlreiche Nachkommen (Gen 15, 1-5). Wie stark der Glaube und die Hoffnung auf einen Neubeginn bei Abram und Sarai inkarniert waren, zeigte sich im „Bund“, den Gott Abram anbot. Ihre Motivation und Entschlossenheit, das Leben nun tatsächlich verändern zu wollen, wird nicht zuletzt durch ihre Namenänderung, worauf Abram nun Abraham und Sarai Sara heißen sollen, zum Ausdruck gebracht ${ }^{16}$.

Als Abraham und Sara doch einen lang ersehnten Sohn, namens Isaak, bekamen ${ }^{17}$, sollte das Familiendrama erst beginnen. Das neugeborene

${ }^{12} \mathrm{Vgl}$ P. Trible, Ominous Beginnings for a Promise of Blessing, in: P. Trible, L. M. Russell (Hrsg.), Hagar, Sarah, and Their Children, Louisville - Kentucky 2006, S. 36.

13 „Als aber der Herr wegen Sarai, der Frau Abrams, den Pharao und sein Haus mit schweren Plagen schlug, ließ der Pharao Abram rufen und sagte: ... Nun, da hast du deine Frau wieder, nimm sie und geh!“. Gen 12, 17-19.

14 „Sarai, Abrams Frau, hatte ihm keine Kinder geboren“. Gen 16, 1a.

${ }_{15}$ Vgl. S. Gerjolj, Živeti, delati, ljubiti: Pedagoška in psihološka interpretacija svetopisemskih pripovedi, Celje 2009, S. 65.

16 „Das ist mein Bund mit dir: Du wirst Stammvater einer Menge von Völkern. Man wird nicht mehr Abram nennen. Abraham (Vater der Menge) wirst du heißen; denn zum Stammvater einer Menge von Völkern habe ich dich bestimmt (Gen 17, 4-5). ... „Weiter sprach Gott zu Abraham: Deine Frau Sarai sollst du nicht mehr Sarai nennen, sondern Sara (Herrin) soll sie heißen (Gen 17, 15).

17 „Der Herr nahm sich Saras an, wie er gesagt hatte, und er tat Sara so, wie er versprochen hatte. Sara wurde schwanger und gebar dem Abraham noch in seinem Alter einen Sohn zu der Zeit, 
„Wunschkind“" stand plötzlich in der Mitte des Geschehens, zuerst bei Sara, bald aber auch bei Abraham. Mit der Geburt Isaaks wollte Sara ihren „Adoptivsohn“ Ismael samt seiner Mutter Hagar loswerden. Die „schmutzige Arbeit" soll Abraham erledigen ${ }^{18}$.

Biblische Geschichten können exegetisch, bibeltheologisch und auch anthropologisch sehr unterschiedlich interpretiert werden. Im Kontext der partnerschaftlichen Dauerbeziehungen symbolisiert Ismael als „uneheliches“ Kind eine „unechte und unaufrichtige“ bzw. eine ,gespielte“ Beziehung, die in Wandlungsprozessen ebenso wichtig ist und im Dienste der echten und aufrichtigen Beziehungen stehen kann. Üblicherweise spiegelt ein jedes „Beziehungsspiel“" zumindest teilweise die Wirklichkeit wider und sich dieses ganz sicher nach der Wahrheit sehnt ${ }^{19}$. Somit ist in Saras Vorschlag, Abraham solle zu Hagar gehen, nicht nur der Wunsch nach einem Sohn bzw. einem „Resultat" des Spiels, sondern auch das Sehnen nach einer unbelasteten und dadurch echten fruchtbaren Beziehung verborgen. Sara bietet Hagar ihrem Mann Abraham nämlich nicht als „Konkubine“, sondern als Frau, $a n^{20}$. Dieses Angebot stellt ausgesprochen die Beziehung und nicht nur die „Früchte“ in den Vordergrund. In der fruchtbaren Beziehung zwischen Abraham und Hagar, die wie „Mann und Frau“ leben, ist also ein tatsächlicher Versuch der echten Kommunikation im Sinne - ,als ob unsere Beziehung echt und unbelastet ist" - zu erkennen. Ismael gibt ihnen somit den „Geschmack“ einer echten Beziehung, obwohl diese vorerst nur „gespielt“ wurde. Erst diese gespielte Beziehung, die gleichzeitig der Versuch eines wahrhaftigen postkathartischen gemeinsamen Lebens ist, motiviert und regt Abraham und Sara an, mit der Klärung ihrer Ehebeziehung fortzusetzen. Es ist aber klar: wenn wir einmal in der partnerschaftlichen Beziehung gelernt haben echt zu kommunizieren, dann ertragen wir kein „Spiel“" mehr und stoßen es $\mathrm{ab}^{21}$.

die Gott angegeben hatte. Abraham nannte den Sohn, den ihm Sara gebar, Isaak“. Gen 21, 1-3.

18 „Eines Tages beobachtete Sara, wie der Sohn, den die Ägypterin Hagar Abraham geboren hatte, umhertollte. Da sagte sie zu Abraham: Verstoß diese Magd und ihren Sohn! Denn der Sohn dieser Magd soll nicht zusammen mit meinem Sohn Isaak Erbe sein“. Gen 21, 9-10.

19 Vgl. S. Gerjolj, Živeti, delati, ljubiti: Pedagoška in psihološka interpretacija svetopisemskih pripovedi, Celje 2009, S. 61.

${ }^{20}$ Sarai, Abrams Frau, nahm also die Ägypterin Hagar, ihre Magd - zehn Jahre, nachdem sich Abram in Kanaan niedergelassen hatte - und gab sie ihrem Mann Abram zur Frau. Gen 16, 3.

${ }^{21}$ Das ist nur eine der möglichen Interpretationen. $\mathrm{Zu}$ wesentlichen anderen Erkenntnissen kommt man, wenn man die Erzählung durch Ismaels Augen liest und reflektiert. Man kann erkennen, dass ihm alle dramatischen und schmerzhaften Erfahrungen zuteilwerden, die oftmals Kinder 


\subsection{Der Wandlungsprozess Abrahams in seiner Vater Rolle}

Die Tatsache, dass die Beziehung zwischen Abraham und Sara zunächst lange Zeit unfruchtbar war, dass sie erst „im Alter“ ihren einzigen Sohn bekamen, der - vor allem von Abraham aus gesehen - als „erglaubtes, erhofftes und erbetetes Kind“" in die Welt kam, bewegte Abraham und Sara nicht nur dahin, für ihr Kind Gott zu danken, sondern auch zum habsüchtigen Angstzustand hin.

Wie die meisten Eltern hatte auch Abraham Angst um sein heranwachsendes und erwachsenwerdendes Kind ${ }^{22}$. Isaaks Prozess des Erwachsenwerdens, daher auch des Selbständig-, Frei- und Anderswerdens erlebte Abraham innerlich äußerst brutal, als wolle ihm Gott auf einem der Berge seinen höchst ersehnten Sohn rauben bzw. ihn sogar - vom eigenen Vater - umbringen lassen ${ }^{23}$.

Auf Grund seiner bisherigen positiven Erfahrungen mit Gott zögerte Abraham bei dieser für ihn existentiellen Herausforderung nicht ${ }^{24}$. Verfolgen wir den „Abraham-Isaak-Weg“ auf den Berg hinauf, so entdecken wir, wie sich der Vater Abraham und der Sohn Isaak in ihrem gespannten Verhältnis stufenweise auf die Beziehung hin konzentrierten. Je näher sie zum Gipfel kamen, desto unbegreiflicher erschienen ihnen auch ihre bisherigen Lebensrollen, die für die beiden in der Art der ,Vater-Kind-Beziehung“ nicht mehr lebbar gewesen waren. Mit der Zeit wurde die Beziehung derartig intensiviert, dass diese vor dem Ende gar nicht mehr in Worte gefasst und artikuliert werden konnte ${ }^{25}$.

aus zwischen den Eltern ungeregelten Verhältnissen ereilen. Auch die Interpretation Hagars, die die Rolle der Mütter in ähnlichen Umstanden erträgt, gehört zu existenziellen Fragen. Geradezu auffallend ist bei ihr die ermutigende Feststellung, dass das Leben sie in der Rolle der Mutter nicht in „unerträgliche Umstände“ bringen kann. Die Liebe zum Kind treibt sie zu heldenhaften Taten und Tugenden, wenn sie auch in ausweglosen Situationen mit Gottes Hilfe eine Lösung findet. Gott steht also auch an ihrer Seite. Vgl. N. J. Cohen, Self, Struggle \& Change: Family Conflict Stories in Genesis and Their Healing Insights for Our Lives, Woodstock 1995, S. 78; M. Pravder Mirkin, The Woman Who Danced By The Sea: Finding Ourselves in the Stories of our Biblical Foremothers, New York 2005, S. 31; P. Trible, Ominous Beginnings for a Promise of Blessing, in: P. Trible, L. M. Russell (Hrsg.), Hagar, Sarah, and Their Children, Louisville - Kentucky 2006, S. 48.

${ }^{22}$ A. Höfer, Gottes Wege mit den Menschen: Ein gestaltpädagogisches Bibelwerkbuch. München 1993, S. 120.

${ }^{23}$ „Gott sprach: Nimm deinen Sohn, deinen einzigen, den du liebst, Isaak, geh in das Land Morija, und bring ihn dort auf einem der Berge, den ich dir nenne, als Brandopfer dar". Gen 22,2.

${ }^{24}$ Vgl. L. Powell, W. R. Self, Holy Murder: Abraham, Isaac, and the Rhetoric of Sacrifice, Lanham - Boulder - New York - Toronto - Plymouth 2007, S. 46.

${ }^{25}$ „Nach einer Weile sagte Isaak zu seinem Vater Abraham: Vater! Er antwortete: Ja, mein Sohn! Dann sagte Isaak: Hier ist Feuer und Holz. Wo ist aber das Lamm für das Brandopfer? Abraham entgegnete: Gott wird sich das Opferlamm aussuchen, mein Sohn. Und beide gingen 
Bevor der Generationskonflikt verarbeitet und gelöst werden konnte, fesselte Abraham noch das letzte Mal seinen Sohn Isaak ${ }^{26}$. Das heißt, als Vater hat er seinen heranwachsenden und erwachsenwerdenden Sohn das letzte Mal als „Kind“" behandelt ${ }^{27}$. In diesem Augenblick sah er ein, mit solchen Taten würde er seinen eigenen Sohn selbst „umbringen“28. Seine „Gewissensstimme“ sagte ihm, Gott wolle doch keine Menschenopfer, sondern vielmehr selbständiges Leben $^{29}$. In der Erkenntnis, mein Kind ist doch nicht mein Eigentum ${ }^{30}$, sondern ein „,von Gott geschenkter Mitmensch“ geschah der Umbruch von einer habituellen „Vater-Kind-Beziehung“ zur partnerschaftlichen „Vater-Sohn-Beziehung ${ }^{\text {“31 }}$.

Nach der „Entbindung“ entdeckte Abraham einen Widder, der sich hinter ihm im Busch verfangen hatte ${ }^{32}$. Der Widder in dieser Erzählung repräsentiert die gehörnten Tiere und verdeutlicht anschaulich das typische, jugendliche

miteinander weiter“ (Gen 22, 7-8). Ab nun an wurde bis Ende ihres gemeinsamen ,Vater-KindWeges" kein Wort mehr gesprochen.

${ }^{26}$ „Als sie an den Ort kamen, den Gott genannt hatte, baute Abraham den Altar, schichtete das Holz auf und fesselte seinen Sohn Isaak und legte ihn auf den Altar, oben auf das Holz". Gen 22, 9.

27 Vgl. J. E. Hartley, New International Biblical Commentary: Genesis, Peabody - Massachusetts - United Kingdom 2000, S. 205.

Die Jugendlichen kennen die Psychologie der Eltern besser als man das denkt. Trotz der wachsenden Wut warten sie darauf, dass sich der Familienraum öffnet. So provozieren sie die Eltern gerne, wenn jemand zu Besuch ist. Die Eltern wollen gute Erzieher sein, den eigenen Kindern gegenüber großmütig und verständnisvoll, denn sie wollen sich vor anderen nicht bloßstellen. Doch weil das „Kind“ provoziert und nicht aufhören will, kann es vorkommen, dass ihnen ,der Kragen platzt“. Dann vergessen sie auf den Besuch und die Umstände und antworten scharf: „So lange du unter meinem Dach lebst, wirst du dich so benehmen, wie ich es will!“ oder etwas Ähnliches. Dieser zwar künstlich inszenierte Vorfall ist der Moment, indem man das Messer gegen das eigene Kind in die Hand nimmt. Ungeachtet des Besuches hat man ihm scharf mitgeteilt, dass es ein „Kind“ ist und dass man es beherrschen kann, wann und wie man es will.

${ }^{28}$,Schon streckte Abraham seine Hand aus und nahm das Messer, um seinen Sohn zu schlachten“. Gen 22, 10.

${ }^{29}$ „Da rief ihm der Engel des Herrn vom Himmel her zu: Abraham, Abraham! Er antwortete: Hier bin ich. Jener sprach: Streck deine Hand nicht gegen den Knaben aus, und tu ihm nichts zuleide! Denn jetzt weiß ich, dass du Gott fürchtest; du hast mir deinen einzigen Sohn nicht vorenthalten. Gen 22, 11-12.

${ }^{30}$ Vgl. R. W. Firestone, J. Catlett, The Ethics of Interpersonal Relationships, London 2009, S. 60 .

${ }^{31}$ Vgl. L. Powell, W. R. Self, Holy Murder: Abraham, Isaac, and the Rhetoric of Sacrifice, Lanham - Boulder - New York - Toronto - Plymouth 2007, S. 79.

${ }^{32}$ Als Abraham aufschaute, sah er: Ein Widder hatte sich hinter ihm mit seinen Hörnern im Gestrüpp verfangen. Abraham ging hin, nahm den Widder und brachte ihn statt seines Sohnes als Brandopfer dar. Gen 22, 13. 
Verhalten. Gehörnte Tiere fühlen sich in einer weichen Umwelt nicht wohl, weil sie ihre Hörner nicht schärfen können. Auch eine zu harte oder zu grobe Umwelt ist nicht gut für sie, denn dort können ihre Hörner abbrechen. Der Busch bietet also gerade genug Widerstand und bedeutet ein gesundes Umfeld für ,gehörnte Tiere“.

Wenn man diese einfache Symbolik auf den Kontext des jugendlichen Heranwachsens überträgt, kann man feststellen, dass eine zu weiche und eine zu harte Erziehung nicht gut sind. Ein guter Erzieher für die Phase des Heranwachsens ist jener, der fähig ist, sich in die Rolle des Busches zu versetzen und diese in der Praxis auch umzusetzen. Im „Gestrüpp“ hat der Widder bzw. der Jugendliche das erste Wort. Er dosiert den Druck und fordert zum Widerstand heraus. Beim Schärfen der Hörner wird niemals der Widder verletzt, sondern immer das Gestrüpp. Wenn den Widder der Widerstand schmerzt, kann er mit dem Schärfen der Hörner aufhören und die Strategie des Kommunizierens verändern. Bei fundamentalen und zentralen Lebens- und Erziehungsfragen, die zu Wandlungsprozessen führen, ist der Mensch häufig offensichtlich erst dann bereit zu lernen, wenn es ,schmerzt“.

\subsection{Integration}

Die Dramatik der Wandlungs- und Integrationsprozesse dauert bis zum Tode der Eltern, Abraham und Sara. Als in der Fremde seine Frau starb, kam er, „um die Totenklage über sie zu halten und sie zu beweinen“ (Gen 23, 2b). Abraham weint also um seine Frau, vielleicht das erste Mal in seinem Leben. Das ganze Leben verarbeitete und klärte er seine eheliche partnerschaftliche Beziehung und schloss seine Arbeit erst am Grabe seiner Frau ab. Als auch er starb, wurde er im selben Grab begraben, von Isaak und Ismael ${ }^{33}$. Somit sind Abraham und Sara, Mann und Frau, endlich beisammen. Ihr Leben lang haben sie sich gesucht, bis sie sich im Grabe endlich finden konnten. Vereint wurden sie durch Ismael und Isaak, durch die gespielte und die echte, wahrhaftig geklärte und deswegen fruchtbare Beziehung.

${ }^{33}$ Er starb in hohem Alter, betagt und lebenssatt, und wurde mit seinen Vorfahren vereint. Seine Söhne Isaak und Ismael begruben ihn in der Höhle von Machpela bei Mamre, auf dem Grundstück des Hetiters Efron, des Sohnes Zohars, auf dem Grundstück, das Abraham von den Hetitern gekauft hatte. Dort sind Abraham und seine Frau Sara begraben. Gen 25,8-10. 


\section{Zweite Generation: die konflikthafte Familie Isaaks}

Der Segen kann gute Voraussetzungen für die kommenden Generationen schaffen, wenn er von einer positiven Lebenseinstellung und der Gewissheit geprägt und unterstützend getragen ist, das Leben zu meistern, Konflikte zu ertragen oder zu lösen, den Lebensfragen und Herausforderungen sinnvoll zu begegnen. Dann kann er Beziehungen stiften und diese kontinuierlich und heilend ,ernähren“. Bei Abraham hingegen sahen wir, dass er nicht nur segnen, sondern auch veruntreuen konnte, dass er ängstlich, egoistisch und habgierig sein konnte. All das gehörte zu seinem „Lebensinhalt“, der bewusst und/oder un/ter/bewusst an die nächsten Generationen weitergegeben, und der zur - gut und auch weniger gut - verdaubaren Nahrung seiner Nachkommen wurde. Die „noch nicht verdauten“ bzw. noch nicht verarbeiteten Lebensinhalte und vor allem lebens- und beziehungsstörenden Gewohnheiten werden - ob wir es wollen oder nicht - vielfach auch zu Herausforderungen der kommenden Generationen.

Wenn auch in einer - im Vergleich zu Abraham - milderen Weise, so schildert uns die Bibel doch eindrucksvoll, wie sehr Isaak - entsprechend seiner vom Vater ,geerbten“ Angewohnheiten - dazu neigte, die Fehler seines Vaters zu wiederholen. Am Beispiel seiner Untreue, die viele Parallelzüge zur Untreuetat seines Vaters aufweist, wird ersichtlich, dass Isaak und seine Nachkommen an der eigenen sowie an der Geschichte ihrer Vorfahren noch lange werden arbeiten müssen, bis diese zu einer reinen Heils-Geschichte werden kann $^{34}$. Denn, die „Geschichte“ ist erst dann verarbeitet, wenn man

${ }^{34}$ Einige Parallelen und Unterschiede hinsichtlich der Un-Treue Isaaks und Abrahams: Ähnlich wie sein Vater fühlte sich Isaak nach einer gewissen Zeit ,ausgehungert“ in seinem Lebensraum: „Im Land brach eine Hungersnot aus, eine andere als die frühere zur Zeit Abrahams“. Gen 26, 1.

${ }^{A u} \mathrm{ch}$ Isaak wollte nach Ägypten. Gott bewahrt ihn allerdings davon: „Geh nicht nach Ägypten hinunter, ...“(Gen 26). Da sich Isaak auch im „Gerar“(Gen 26, 1b), einem im Vergleich zu Ägypten weniger verlockend befremdeten Land, doch „,bedroht fühlte“, war er, wie sein Vater, ohne weiteres bereit, seine Frau zu „verkaufen“: „Als sich die Männer des Ortes nach seiner Frau erkundigten, sagte er: Sie ist meine Schwester. Er fürchtete sich nämlich zu sagen: Sie ist meine Frau. Er dachte: Die Männer des Ortes könnten mich sonst wegen Rebekka umbringen. Sie war nämlich schön“ (Gen 26, 7). Dank der Tatsache, dass sich er und Rebekka in ihrer partnerschaftlichen Beziehung weiterhin verwirklichen konnten, konnte er vom Vollzug seiner Untreue verschont bleiben: „Nachdem er längere Zeit dort zugebracht hatte, schaute einmal Abimelech, der König der Philister, durch das Fenster und sah gerade, wie Isaak seine Frau Rebekka liebkoste. ... Wie konntest du behaupten, sie sei deine Schwester? ... Beinahe hätte einer der Leute mit deiner Frau geschlafen“. Gen 26, 8-10. 
unter ähnlichen Bedingungen und bei ähnlichen Herausforderungen die Untaten der Vorfahren nicht wiederholt, sondern „es besser macht“.

Die von ihren Vorfahren ,geerbten“ und aus der Vergangenheit mitgebrachten Belastungen machten sich im Leben Isaaks und in dem seiner Familie tiefgreifend bemerkbar. Erstaunlicherweise schildert uns die Bibel, dass auch Isaaks Frau Rebekka lange Zeit unfruchtbar war ${ }^{35}$. Dass allerdings einiges von Abrahams und Saras Untreue und ein Teil der sonstigen beziehungsbelastenden Lebensinhalte bis zur Gründung der Familie Isaaks bereits verarbeitet werden konnten, lässt sich unter anderem erkennen, indem die Ehebeziehung Abrahams bedeutend länger unfruchtbar als war die Isaaks ${ }^{36}$. Weiterhin war die Ehebeziehung Isaaks im Vergleich zur jener Abrahams fruchtbarer. Als Rebekka nämlich dann doch schwanger wurde, gebar sie Zwillinge.

Mehr Leben bringt aber auch neue dramatische Szenen mit sich. Die unverarbeiteten Spannungen und Konflikte der Eltern und Großeltern bekamen die Zwillingssöhne Isaaks, Esau und Jakob, bereits im Mutterleib zu spüren. Die beiden standen bereits vor ihrer Geburt miteinander in Konflikt und bekamen somit keine gute Startposition für ihr Leben ${ }^{37}$.

Der bereits im Mutterschoß aufgebrochene „Brüderkonflikt“" wurde mit der Geburt Esaus und Jakobs keinesfalls beendet. Ganz im Gegenteil, dieser artete zunehmend in ständige Rivalität zwischen den Zwillingsbrüdern aus. Der biblische Text weist eindeutig darauf hin, dass Esau das Lieblingskind Isaaks war, während Rebekka Jakob lieber hatte ${ }^{38}$. Die Vorliebe der Eltern für je ein Kind - vor allem bei sogenannten Zweikinderfamilien - lässt oft auf Rivalität zwischen Vater und Mutter schließen. Allzu oft werden partnerschaftliche Ehekonflikte und Rivalitäten spiegelhaft auf die Kinder/ rollen projiziert und im Rahmen einer Geschwisterrivalität ausgetragen. Auch in vielen Familien heutiger Zeit stecken hinter konfliktreichen Rivalitäten archetypische Rollensysteme, wobei es auf beiden Konfliktseiten um Verteidigung oder Eroberung besserer Machtpositionen geht, und wo

${ }^{35}$ „Isaak betete zum Herrn für seine Frau, denn sie war kinderlos geblieben, und der Herr ließ sich von ihm erbitten“. Gen 25, 21.

${ }^{36}$ „Abraham war hundert Jahre alt, als sein Sohn Isaak zur Welt kam“. Gen 21, 5.

„Isaak war sechzig Jahre alt, als sie“ (Esau und Jakob) „geboren wurden“. Gen 25, 26c.

${ }^{37}$ „Als seine Frau Rebekka schwanger war, stießen die Söhne einander im Mutterleib“. Gen 25, 21b-22a.

${ }^{38}$ „Isaak hatte Esau lieber, denn er aß gern Wildbret; Rebekka aber hatte Jakob lieber“. Gen 25, 28. 
des Öfteren das „patriarchalische Recht“ und die „matriarchalische List“ aufeinanderprallen ${ }^{39}$.

Der vom Vater verwöhnte Esau wird Jäger, was eine ausgesprochen männliche Tätigkeit und männliche Weltauffassung war ${ }^{40}$. Er wird „ganz der Papa“. Sittenlehre, Tradition und ethisch-religiöse Werte spielten für ihn keine bedeutende Rolle ${ }^{41}$. Selbst auf sein patriarchalisches Recht des Erstgeborenen, das sehr wohl auch materielle Privilegien beinhaltet ${ }^{42}$, war er bereit zu verzichten, bloß um ,satt" zu werden ${ }^{43}$. Dieses sollte ohnedies vom Vater weitergegeben worden sein.

Jakob dagegen war eher ein „weiblicher Menschentyp“, folgsam und sorgsam, aber auch zielstrebig und „listig ${ }^{644}$. Als „Fersenhalter“ verstand er die vitalen Schwächen seines Bruders auszunützen, um sich eine bessere „Machtposition“ zu verschaffen. So wusste er seinen Bruder in der „Hungersnot" kaltblütig, schlau und listig auszutricksen ${ }^{45}$.

Der Familienkonflikt konnte ertragen werden, bis der Zeitpunkt eintraf, wo Lebensentscheidungen getroffen werden mussten. Es war ohnedies zu spät, denn Vater Isaak war bereits „blind“446. Es scheint nur die Mutter Rebekka die Familienverhältnisse richtig verstanden zu haben. So wusste sie die konfliktreiche Familienkoalition auch dementsprechend zu steuern. Sie

${ }^{39} \mathrm{Vgl}$. A. Höfer, Gottes Wege mit den Menschen: Ein gestaltpädagogisches Bibelwerkbuch, München 1993, S. 133.

40 „Esau war ein Mann geworden, der sich auf die Jagd verstand, ein Mann des freien Feldes“. Gen 25, 27.

${ }^{41}$ Vgl. J. E. Hartley, New International Biblical Commentary: Genesis, Peabody - Massachusetts - United Kingdom 2000, S. 247.

${ }^{42} \mathrm{Vgl}$. J. H. Walton, The NIV Application Commentary: From biblical text ... to contemporary life, Grand Rapids - Michigan 2001, S. 550.

43 „Da schwor er ihm und verkaufte sein Erstgeburtsrecht an Jakob. Darauf gab Jakob dem Esau Brot und Linsengemüse; er aß und trank, stand auf und ging seines Weges. Vom Erstgeburtsrecht aber hielt Esau nichts“. Gen 25, 33b-34.

${ }^{44}$ Vgl. N. J. Cohen, Self, Struggle \& Change: Family Conflict Stories in Genesis and Their Healing Insights for Our Lives, Woodstock 1995, S. 98.

45 „Einst hatte Jakob ein Gericht zubereitet, als Esau erschöpft vom Feld kam. Da sagte Esau zu Jakob: Gib mir etwas zu essen von dem Roten, ... Jakob gab zur Antwort: Dann verkauf mir jetzt sofort dein Erstgeburtsrecht! Schau, ich sterbe vor Hunger, sagte Esau, was soll mir da das Erstgeburtsrecht? Jakob erwiderte: Schwor mir jetzt sofort! Da schwor er ihm und verkaufte sein Erstgeburtsrecht an Jakob“. Gen 25, 29-33.

${ }^{46}$ „Als Isaak alt geworden und seine Augen erloschen waren, so dass er nicht mehr sehen konnte, rief er seinen älteren Sohn Esau“. Gen 27, 1a. 
wartete ab und, als der Zeitpunkt kam, da lauschte sie und bekam das entscheidende Gespräch zwischen dem Vater und seinem Lieblingssohn Esau $\mathrm{mit}^{47}$. Als Esau von seinem blinden Vater auf die Jagd geschickt wurde, um „ein leckeres Mahl“" zu bereiten, nachdem er den Segen des Vaters bekommen sollte $^{48}$, brachte sie ihren Jakob ins Spiel. Wenn auch hier die „Vater-Mutter-Rivalität" nicht auszuschließen ist ${ }^{49}$, war sie jedenfalls bereit, alles für ihren Lieblingssohn zu tun und auch alles über sich ergehen zu lassen ${ }^{50}$. Sie übernahm das Wort und die Verantwortung, Jakob musste nur noch „normal“ funktionieren. Sie bereitete das „leckere Mahl“, zog Jakob „esaumäßig“ an, ermutigte ihn, redete ihm gut zu und schickte ihn schließlich zum Vater, wo er dann seine (und insofern es sich hier um elterliche Rivalität handelt, auch ihre) Rolle sicher und selbstbewusst fertigspielte (Gen 27, 5-29). Seine und der Mutter List und Lügen waren erfolgreich. Er bekam den Segen und wurde zum „Mächtigsten“ in der Familie bestimmt.

Als die „Mutter-Jakob-Koalition“ die „Vater-Esau-Koalition“ überlistete, war der Familienkonflikt keinesfalls gelöst. Esau besaß zwar keine „,institutionelle“ Macht, wollte aber bis zum Tode seines Vaters warten, um dann durch eine „Familienrevolution“ an die Macht zu gelangen, indem er seinen Bruder umbringen und somit die herrschende „matriarchalische“ Koalition entmachten würde ${ }^{51}$. Nun, auch diesmal spielte Rebekka ihre Rolle mit großer Sensibilität weiter und verhinderte es, indem sie Jakob zu ihrem Bruder Laban schickte ${ }^{52}$. Der Konflikt wird also nicht gelöst, sondern bloß verschoben.

47 „Rebekka hatte das Gespräch zwischen Isaak und seinem Sohn Esau mit angehört“. Gen 27, 5a.

48 „Nimm jetzt dein Jagdgerät, deinen Köcher und deinen Bogen, geh aufs Feld, und jag mir ein Wild! Bereite mir dann ein leckeres Mahl, wie ich es gern mag, und bring es mir zum Essen, damit ich dich segne, bevor ich sterbe“. Gen 27, 3-4.

${ }^{49} \mathrm{Vgl}$. A. Höfer, Gottes Wege mit den Menschen: Ein gestaltpädagogisches Bibelwerkbuch, München 1993, S. 134.

${ }^{50}$ „Seine Mutter entgegnete: Dein Fluch komme auf mich, mein Sohn“. Gen 27, 13a.

51 „Esau war dem Bruder feind wegen des Segens, mit dem ihn sein Vater gesegnet hatte, und Esau sagte: Es näheren sich die Tage der Trauer um meinen Vater; dann werde ich meinen Bruder Jakob umbringen“. Gen 27, 41.

52 „Als man Rebekka hinterbrachte, was ihr ältester Sohn gesagt hatte, ließ sie Jakob, ihren jüngeren Sohn, rufen und sagte zu ihm: Dein Bruder Esau will sich an dir rächen und dich töten. Nun aber, mein Sohn, hör auf mich! Mach dich auf und flieh zu meinem Bruder Laban nach Haran!“. Gen 27, 42-43. 


\section{Dritte Generation: Jakob und seine heilende Beziehungen}

Wie oben ersichtlich, ergriff Jakob aus Angst vor seinem Bruder Esau die Flucht und machte sich auf den Weg zu Laban, dem Bruder seiner Mutter. Bereits unterwegs zu Laban begegnete Jakob dessen Tochter Rahel und er verliebte sich in sie. Als er bei Laban ankam, hatte Rachel bereits von ihm erzählt. Er wollte bleiben und ihm dienen, was Laban gerne annahm (Gen 29, 1-14). Als Jakob von Laban nach dem Lohn für seinen Dienst gefragt wurde, schlug er vor, sieben Jahre für die jüngere Tochter Rahel zu dienen ${ }^{53}$.

„Laban hatte zwei Töchter; die ältere hieß Lea, die jüngere Rahel. Die Augen Leas waren matt, Rahel aber war schön von Gestalt und hatte ein schönes Gesicht" (Gen 29, 16-17).

Obwohl der Vorschlag Jakobs nicht ganz den damaligen Sitten (Alter vor Schönheit) entsprach ${ }^{54}$, erklärte sich Laban damit doch einverstanden ${ }^{55}$. So „diente Jakob also um Rahel sieben Jahre. Weil er sie liebte, kamen sie ihm wie wenige Tage vor" (Gen 29, 20). Nachdem nun für Jakob die Zeit des Dienens um Rahel abgelaufen war, wollte er sie erwartungsgemäß als Frau haben ${ }^{56}$. Laban zögerte allerdings und wollte zuerst seine ältere Tochter verheiratet haben. So ging Jakob nach dem „Polterabend“"57 zu Bett, in Erwartung, Vater Laban - wie versprochen - werde ihm die nun „verdiente“ Frau Rahel bringen. Laban brachte aber nicht die ersehnte Schönheit Rahel zu ihm, sondern ihre unschöne ältere Schwester Lea ${ }^{58}$, was Jakob allerdings erst am Morgen bemerkte ${ }^{59}$.

53 „Jakob hatte Rahel lieb, und so sagte er: Ich will dir um die jüngere Tochter Rahel sieben Jahre dienen“. Gen 29, 18.

${ }^{54}$ Vgl. E. Schirmer, Müttergeschichten: Frauen aus dem Alten Testament erzählen von ihrem Leben, Offenbach 1989, S. 22.

55 „Laban entgegnete: Es ist besser, ich gebe sie dir als einem anderen. Bleib bei mir!“. Gen 29, 19.

56 „Dann aber sagte er zu Laban: Gib mir jetzt meine Frau; denn meine Zeit ist um, und ich will nun zu ihr gehen“. Gen 29, 21.

57 „Da ließ Laban alle Männer des Ortes zusammenkommen und veranstaltete ein Festmahl“. Gen 29, 22.

58 „Am Abend nahm er aber seine Tochter Lea, führte sie zu ihm, und Jakob wohnte ihr bei“. Gen 29, 23.

59 „Am Morgen stellte sich heraus: Es war Lea“. Gen 29, 25 a. 
In diesem Moment ging dem wütenden Jakob einiges durch den Kopf. Er fühlte sich von Laban hintergangen ${ }^{60}$.Alsihm Labandanndieallgemeingültigen Gewohnheiten, Bräuche und Sitten erklärte, hatte er keine andere Wahl, als es zu akzeptieren, wollte er ja doch (auch) Rahel heiraten ${ }^{61}$. Er musste innerlich eingestehen, dass der Betrug zu seiner bisherigen Lebenspraxis gehörte, und wenn man den Betrug zum Lebensstil und Handlungsprinzip macht, dann kann dieser auch zum Bumerang werden.

Jakob lernte bei seiner Mutter jedoch zu „kämpfen“ und dachte überhaupt nicht an Kapitulation. Laban gab ihm demnach eine „zweite“ Chance, auch Rahel als Ehefrau zu bekommen. Allerdings musste er zuerst mit Lea die Brautwoche verbringen und anschließend versprechen, für Rahel weitere sieben Jahre zu dienen ${ }^{62}$.

Im Hinblick aufdie tiefenpsychologische Archetypologie sowie hinsichtlich der ganzheitlichen und mehrschichtigen Bibelinterpretationen lässt sich hier eine beziehungsverarbeitende und vergangenheitsbewältigende Lebensfrage herausfinden, die lauten könnte: „Was gibt uns die Geschichte, wenn hinter den zwei unheimlich verschiedenen Gestalten und Gesichtern eine Person steckt; wenn sich eine Person innerhalb kürzester Zeit total verändern kann und sich in einer Nacht von einer schönen, attraktiven Frau mit kristallklaren Augen zu einer abstoßenden, mattäugigen und krummen Figur verwandelt?“ Dann können einige Erkenntnisse und Konsequenzen vor allem hinsichtlich partnerschaftlicher Beziehungen ans Licht gebracht werden.

Diese biblische Geschichte bringt unheimlich feinfühlig und tiefgreifend zum Ausdruck, wie wichtig und beglückend es ist, die Ehefrau bzw. den Ehemann nicht besitzen zu wollen und ihr/ihm achtungsvoll und partnerschaftlich zu begegnen. Unabhängig von den damaligen Traditionen und Bräuchen stand beim Aufbau der partnerschaftlichen Beziehung zwischen Jakob und Rahel eine achtsam dienende und beinahe zeitüberschreitende

60 „Da sagte Jakob zu Laban: Was hast du mir angetan? Habe ich dir nicht um Rahel gedient? Warum hast du mich hintergangen?". Gen 29, 25b-c.

${ }^{61}$ „Laban erwiderte: Es ist hierzulande nicht üblich, die Jüngere vor der Älteren zur Ehe zu geben. Verbring mit dieser noch die Brautwoche, dann soll dir auch die andere gehören um weitere sieben Jahre Dienst“". Gen 29, 26-27.

62 ,Verbring mit dieser noch die Brautwoche, dann soll dir auch die andere gehören um weitere sieben Jahre Dienst. Jakob ging darauf ein... Er blieb noch weitere sieben Jahre bei Laban im Dienst". Gen 29, 20. 
„glückliche“ Liebe im Vordergrund ${ }^{63}$. Man kann allerdings davon ausgehen, dass sich in jenem Augenblick, als Jakob zu Laban kam und ihm mitteilte, die „dienende Zeitperiode“ sei nun vorbei, von nun an „gehöre Rahel mir“, ich will sie nun „haben“, der Charakter der Beziehung zu ihr schlagartig veränderte. Von einer liebevoll dienenden wurde Jakob zur habgierigen und herrschenden Person. Jakob zeigte die Kehrseite, also sein „wahres“ Gesicht. Dies machte sich auch an der Gestalt Rahels sowie an ihrem Gesichtsausdruck und ihrem Blick bereits ,in der ersten Nacht“, d. h. schnell und noch unter seinem „Liebesrausch“ bemerkbar, was sich allerdings erst am Morgen, $d$. $h$. bei seinem bewussten Anblick herausstellte (Gen 29, 21-25). Jakob heiratete also nicht nur Rahel, sondern auch ihre „Kehrseite“, die Lea hieß. Sie ist sogar älter, lebte aber während der Jahre des Sich-in-Liebe-Kennenlernens und Sich-einander-Näherkommens weit im Hintergrund. Mit ersten Beziehungskonflikten zeigte somit auch Rahel ihr ,wahres“ Gesicht.

Wie bei vielen Partnerschaften könnte auch bei Jakob und Rahel nach einer solchen Erfahrung, wo sich mehr oder weniger beide Partner betrogen fühlen, indem sie meinten, einen ,anderen“ Menschen geheiratet zu haben, die Beziehung zerbrechen. Dem wurde aber nicht so. Jakob wollte bekannterweise unbedingt Rahel heiraten. Vorerst musste er aber Lea, d. h. die Schattenseiten seiner Partnerin, akzeptieren. Er tat es auch. Dann begab er sich gleich auf die Suche nach Rahel, indem er seine zweite „Dienstzeitperiode“ einschlug ${ }^{64}$. Erstaunlicherweise musste er diesmal auf Rahel keine sieben Jahre warten, sondern sie wurde ihm unmittelbar nach der Brautwoche gegeben ${ }^{65}$. In jenem Augenblick, als Jakob wiederum versuchte, seine Frau partnerschaftlich, dienend und liebevoll $\mathrm{zu}$ begegnen, meldete sich hinter den Gestalt- und Charakterzügen Leas wiederum die liebenswürdige Rahel.

Das tatsächlich wahre Gesicht zeigte sich also bei beiden Lebenspartnern erst während der zweiten Dienstperiode. Denn, wie Jakob weder ein ausschließlich liebend dienender noch ein ausschließlich possessiv herrschsüchtiger Mensch war, sondern potentiell beides davon in ihm

63 „Jakob diente also um Rahel sieben Jahre. Weil er sie liebte, kamen sie ihm wie wenige Tage vor". Gen 29, 20.

${ }^{64}$ Man darf dabei nicht die symbolische Botschaft der Zahl sieben vergessen, die Vollkommenheit und Überfluss bedeutet. Jakobs Dienst bedeutet somit die Wandlung der erst gescheiterten liebenden Einstellung und Lebenshaltung, die nun nicht mehr endet.

${ }^{65}$ „Er verbrachte mit Lea die Brautwoche, dann gab ihm Laban seine Tochter Rahel zur Frau... Er blieb noch weitere sieben Jahre bei Laban im Dienst“. Gen 29, 29-30. 
steckte, so war auch seine Lebenspartnerin weder ausschließlich Rahel noch ausschließlich Lea, sondern sowohl Rahel als auch Lea. Wenn wir - was die biblische Ausdrucksweise ohne weiteres ermöglicht - eine dahinterstehende Person zu entdecken versuchen, so kommen wir zur Einsicht, dass man eine Rahel ohne Lea gar nicht heiraten kann, denn sie gehören existenziell zusammen und sind in einem jeden Lebenspartner zu entdecken. Wie Rahel und Lea in einer Person, so hat jeder Mensch eine sonnige und bejahte und eine dunkle und verdrängte Seite. Die Schattenseite kommt oft erst nach der Hochzeit ans Tageslicht und wird erst später zunehmend aktiviert. So ist jede Hochzeit auch eine „Schattenhochzeit"“66.

Wenn Jakob erst Lea als Vertreterin der Schattenseite akzeptiert, so heißt es nicht, dass er sie in seiner partnerschaftlichen Beziehung bloß „duldet“. Vielmehr geht es darum, dass - wenn Jakob Rahel auch mehr liebt als Lea - er auch Lea liebt ${ }^{67}$. Das heißt, er lernte auch die Schattenseiten seiner Frau zu lieben, was nicht zuletzt in der großen Fruchtbarkeit Leas zum Ausdruck kommt ${ }^{68}$. In einer liebenden und stabilen Ehebeziehung werden die Schattenseiten einer Person geheilt und werden sogar fruchtbarer als die sonnigen und von Anfang an bejahten Seiten ${ }^{69}$.

\subsection{Jakobs Versöhnung mit sich selbst}

Jakob lernte in seiner dauerhaften Ehebeziehung Konflikte zu ertragen und diese je nach Möglichkeit zu lösen. Nun stand ihm noch sein Bruderkonflikt bevor. Nachdem er sich von seinem Schwiegervater trennte, konnte er den Konflikt mit seinem Bruder nicht mehr ertragen. So begab er sich auf den Weg, um sich auch mit ihm zu versöhnen. Davor musste er allerdings noch mit sich selbst zurechtkommen, worüber uns die Bibel in der Geschichte über Jakobs Kampf mit Gott berichtet (Gen 33, 23-33).

${ }^{66}$ A. Höfer, Gottes Wege mit den Menschen: Ein gestaltpädagogisches Bibelwerkbuch, München 1993, S. 136-137.

${ }^{67}$ Vgl. M. Pravder Mirkin, The Woman Who Danced By The Sea: Finding Ourselves in the Stories of our Biblical Foremothers, New York 2005, S. 83.

${ }^{68}$ Lea hatte sieben Kinder (sechs Söhne und eine Tochter), Rahel dagegen nur zwei (Josef und Benjamin). Gen 35, 23-24.

${ }^{69}$ Vg. J. P. Forgas, Affective Influences on Communication and Attribution in Relationships, in: V. Manusov, J. H. Harvey (Hrsg.), Attribution, Communication Behavior, and Close Relationships, Cambridge - New York - Melbourne - Madrid - Cape Town - Singapore - Sao Paulo - Delhi - Tokyo - Mexico City 2001, S. 12. 


\section{4}

Der biblischen Erzählung nach war Jakob unter anderem auch ein »Betrüger«, was er in der angedeuteten Geschichte über seinen Kampf mit Gott auch eingestehen musste. Hinter „einem Mann“, der sich in einen Engel und sogar in Gott verwandelt, verbirgt sich also seine sündhafte Vergangenheit, das heißt die Schattenseite seiner Persönlichkeit ${ }^{70}$. Er begibt sich in den Kampf mit ihr und zeitweise erscheint es ihm, als wäre es unmöglich, sie anzunehmen. Seine Vergangenheit erscheint stark wie Gott und er fürchtet sich, dass sie ihn bezwingen und zerstören würde. Manchmal schämt man sich der eigenen Taten und will sich diese nicht eingestehen, doch gerade deswegen wiederholt man sie immer wieder aufs Neue. Man fürchtet sich, das Eingeständnis könnte einen zerstören, man würde vor sich selbst zu Grunde gehen. Insbesondere ein öffentliches Eingeständnis könnte uns unterkriegen, das Selbstbild ersticken und den „guten Ruf“ ruinieren. Jakob tröstete sich bis zu diesem Tag mit solchen und ähnlichen Lügen. Nun erkennt er endlich, dass es ohne ein Annehmen und Bekennen von Sünden und Fehlern kein Verzeihen und keine Veränderung gibt ${ }^{71}$.

Jakob muss zuerst sich selbst, und dann auch der Familie die Wahrheit über die Vergangenheit erzählen. Dies wird ihn verletzen und ,das Hüftgelenk ausrenken" (Gen 32, 26). In den Augen der Familienmitglieder und in der Öffentlichkeit wird er nicht mehr fehlerlos und vollkommen, sondern verletzt, verletzlich und verkrüppelt sein. Sein Bild wird stark „,hinken“. Doch trotzdem kämpft er die ganze Nacht, bis zur Morgenröte bzw. bis er einen klareren und deutlicheren Blick in die Zukunft verspürt. Erst dann lässt die Vergangenheit nach und kündigt mit folgenden Worten an aufzugeben: „Lass mich los; denn die Morgenröte ist aufgestiegen (Gen 32, 27).

Doch Jakob lässt nicht nach. Er fühlt sich zunehmend stärker und will den Kampf zu Ende führen. Er will die Vergangenheit nicht nur eingestehen und sie als eigene annehmen, sondern er erwartet von ihr sogar einen Segen: „Ich lasse dich nicht los, wenn du mich nicht segnest" (Gen 32, 27b). Es geht also nicht nur darum, die unverfälschte und dunkle Vergangenheit vorbehaltlos anzunehmen und sie vor sich selbst und den anderen einzugestehen, sondern er ist sogar dankbar für sie, denn er lernt aus ihr und erwartet von ihr eine

${ }^{70}$ Vgl. J. E. Loder, The Logic of the Spirit: Human Development in Theological Perspective, San Francisco 1998, S. 302.

${ }^{71}$ Vgl. M. A. Corey, Job, Jonah, and the Unconscious: A Psychological Interpretation of Evil and Spiritual Growth in the Old Testament, Lanham - New York - London 1995, S. 9. 
große Fruchtbarkeit. Er verbirgt seine sündige Vergangenheit nicht, sondern rühmt sich ihrer, denn in ihrem Angesicht treten die Früchte der Veränderung stärker hervor, gleichzeitig aber erhalten das Eingestehen und das Rühmen die Sensibilität der Nächsten aufrecht, die schnell reagieren würden, wenn sich Anzeichen des Rückfalles in die Sünden der Vergangenheit bemerkbar machen würden. Erst nachdem er es bewusst annimmt und integriert, daraus lernt und in Form der Transformation die ersten Früchte erhält, kann Jakob entspannt und gesegnet aufatmen ${ }^{72}$.

Interessant ist, wie ihn die Vergangenheit gegen Ende des Kampfes prüft und nach dem Namen fragt. Jakob stellen sich die Fragen: Worin liegt eigentlich der Sinn all dieses Kämpfens und dieser Pein? Lohnt es sich? Um wessen Willen? u. ä. Hinter der Frage nach dem Namen verbirgt sich also das Herausfordern des Sinnes und der Zweifel daran ${ }^{73}$. Letzterer tritt gerade in Momenten der Schwäche und der Erschöpfung auf, denn der „Kampf“ will ganz einfach nicht enden. Trotz der Erschöpfung festigt gerade dieses Herausfordern Jakob in der Beharrlichkeit und Aufrichtigkeit, denn ohne Bedenken gibt er Antwort und sagt, er sei „Jakob“ - der Fersenhalter, Schwindler und Betrüger ${ }^{74}$. Mit dem Bekenntnis des Namens stellt er sich vor den Spiegel und blickt in die eigenen Augen. Ohne zu zaudern gesteht und akzeptiert er den eigenen „Zusammenbruch“, aus dem sofort ein neuer Mensch zu wachsen beginnt: „Nicht mehr Jakob wird man dich nennen, sondern Israel; denn mit Gott und Menschen hast du gestritten und du hast gewonnen“ (Gen 32, 29). Jakob muss also tatsächlich als Schwindler und Betrüger zusammenbrechen, um daraus als neuer Mensch mit dem Namen Israel zu erwachsen, der Kraft, Kommunikation und Gemeinwesen ausstrahlt. Israel als „Kämpfer mit Gott“75 drückt im Kontext der edukativ-psychologischen Interpretation auch die Fähigkeit zur Gestaltung einer eigenen Identität aus; er besiegt „Gott“ nämlich damit, dass er ihn als ,zweite“, dunkle und sündhafte Seite der Vergangenheit in das Persönlichkeitswachstum integriert. Indem

${ }^{72}$ A. L. Kille, Jacob: A Study in Individuation, in: David L. Miller (Hrsg.), Jung and the Interpretation of the Bible, New York 1995, S. 51.

${ }^{73} \mathrm{Vgl}$. A. Höfer, Gottes Wege mit den Menschen: Ein gestaltpädagogisches Bibelwerkbuch, München 1993, S. 138.

${ }^{74} \mathrm{Vgl}$. J. E. Hartley, New International Biblical Commentary: Genesis, Peabody - Massachusetts - United Kingdom 2000, S. 236.

${ }^{75}$ Vgl. J. E. Hartley, New International Biblical Commentary: Genesis, Peabody - Massachusetts - United Kingdom 2000, S. 284. 
Jakob seinen „Jakob“ annimmt, verwandelt er sich vom „,vollkommenen“, egoistischen, selbstverliebten und verschlossenen Schwindler und Betrüger $\mathrm{zu}$ einem unvollkommenen und verletzbaren, doch gleichzeitig starken, kommunikativen und sozialen Menschen.

Interessanterweise verwendet die Bibel ab nun an in den weiteren Texten beide Namen, Jakob und Israel, was verdeutlicht, wie schwierig es ist, sich tatsächlich und endgültig zu verändern bzw. wie sehr man auch nach grundlegenden Veränderungen noch immer vom Rückfall in die Vergangenheit gefährdet ist ${ }^{76}$. Wenn er nämlich dazu neigt, alte Sünden zu begehen, wird er Jakob genannt, wenn er aber als Integrationsfigur sowie offene und glaubende Kommunikationspersönlichkeit identifiziert werden kann, trägt er den Namen Israel.

Vor dem Ende des Kampfes verlangt auch Jakob: „Nenne mir doch deinen Namen!?“ (Gen 32, 30), wodurch die Langwierigkeit des Kampfes mit der Vergangenheit und die Unnachgiebigkeit verdeutlicht werden sowie das wiederholte Aufflammen von Gefühlen, die den Zweifel an den Veränderungsbestrebungen aufkommen lassen. Es ist schwierig zur endgültigen Antwort und zum letzten Sinn zu gelangen, und dieser weicht uns immer wieder aufs Neue aus. Auch Jakob bekommt nämlich keine Antwort, sondern nur eine weitere herausfordernde und mit Zweifeln verbundene Frage: „Was fragst du mich nach meinem Namen?" (Gen 32, 30b).

Anstatt einer Antwort bekommt er den Segen (Gen 32, 30c), woraus geschlossen werden kann, dass es wichtiger ist, die Früchte des geänderten Lebens zu bewerten, als immer wieder aufs Neue nostalgisch den Namen bzw. die Gründe und die Schuldigen für die ,verfehlte Vergangenheit zu suchen“. In diesem Sinne geht die Heilige Schrift über die Analyse hinaus, die per definitionem in der Vergangenheit verhaftet bleibt. Die Heilige Schrift „analysiert" die Vergangenheit nur so lange, bis sie uns aus dieser lehren kann, dann aber regt sie uns an, sie in allen Dimensionen anzunehmen und ,in Ruhe zu lassen“, damit sie in uns absterben und sich zum „Humus“ verwandeln kann, aus dem starke und feste Entscheidungen erwachsen können.

Jakob kam verletzt und verkrüppelt aus diesem Kampf hervor, doch er ist trotzdem fähig, den Fluss zu durchschreiten und den entscheidenden Schritt in die Zukunft zu setzen. Er hat sich endlich mit sich selbst versöhnt und braucht

${ }^{76}$ Vgl. N. J. Cohen, Voices from Genesis: Guiding Us Through the Stages of Life, Woodstock, Vermont 1998, S. 83. 
sich nicht mehr zu verbergen und zu verstellen. Er weiß jetzt auch, dass die ganzheitliche und ungeteilte Annahme von sich selbst die Bedingung sowohl für den Ausdruck als auch für das Heilen des Lebens und der Beziehungen ist $^{77}$. Endlich getraut er sich vor sich selbst, vor Gott und vor der Familie so zu sein, wie er in Wahrheit ist: Jakob, der sich verändert und zu Israel wird.

Der biblische Bericht über Jakobs Kampf mit Gott simuliert also einen intrapsychischen Lernprozess, zu dem ein jeder (selbst)erziehungsfähige Mensch herausgefordert ist. In den meisten Fällen sind nämlich zwischenmenschliche Spannungen Folge der nicht bewältigten intrapersonalen innerlichen Konflikte und dort, an den Wurzeln, gehören sie auch bereinigt ${ }^{78}$.

\subsection{Die Versöhnung Jakobs mit seinem Bruder Esau}

„Versöhnung unter Geschwistern oder Ehepartnern braucht oft viel Zeit, viel schrittweises Entgegenkommen, viel Vorschuss an Vertrauen, viel eigene Reue und Wandlung “79. Vieles davon konnte Jakob bereits erbringen. Als Vorschuss und Zeichen des Vertrauens, das nach wie vor von Angst und Zweifel geprägt wurde, ließ er sich von seiner Familie und anderen Vertrauenspersonen zu seinem Bruder begleiten.

Jakob kannte seinen ,alten“ Bruder und wusste nicht, wie dieser auf sein Erscheinen reagieren würde. So teilte er vorsichtshalber seine Familie in drei Gruppen auf, um schlimmstenfalls bei einem Wutausbruch seines Bruders nicht gleich die gesamte Familie zu verlieren ${ }^{80}$. Der Mut, das Vertrauen und die Demut einerseits sowie die Angst und Verzweiflung Jakobs andererseits werden aber seinen Bruder Esau beeindruckt haben und bereiteten ihn auf die ergreifende und tiefbewegende Versöhnungsbegegnung vor. Dieses vertrauensvolle schrittweise Entgegenkommen entmachtete Esau und er kam zu Jakob ohne Gewalt und versöhnungsbereit ${ }^{81}$.

${ }^{77}$ Vgl. H. Cloud, J. Townsend, How People Grow: What the Bible Reveals about Personal Growth, Grand Rapids - Michigan 2001, S. 153.

${ }^{78}$ Vgl. R. D. Bell, Biblical Models of Handling Conflict, Vancouver 2001, S. 48.

${ }^{79}$ A. Höfer, Gottes Wege mit den Menschen: Ein gestaltpädagogisches Bibelwerkbuch, München 1993, S. 138.

${ }^{80}$ „Jakob blickte auf und sah: Esau kam mit vierhundert Mann. Da verteilte er die Kinder auf Lea und Rahel und auf die beiden Mägde. Die Mägde und deren Kinder stellte er vorn hin, dahinter Lea und ihre Kinder und zuletzt Rahel und Josef". Gen 33, 1-2.

${ }^{81}$ „Er trat vor und warf sich siebenmal zur Erde nieder, bis er vor seinem Bruder stand. Esau lief ihm entgegen, umarmte ihn und fiel ihm um den Hals; er küsste ihn, und sie weinten“". Gen 33, 3-4. 
Es hat den Anschein, dass mit dem versöhnlichen Umarmen und Weinen der Versöhnungsprozess vollendet gewesen wäre. Dem ist aber nicht so. Zuerst schließen sich der Reuetat Jakobs sämtliche Familienmitglieder an, was auf eine starke schicksalhafte Identifizierung mit ihrem Ehepartner und Vater sowie auf familiäres gemeinschaftliches „Wir-Gefühl“ hindeutet ${ }^{82}$. Zudem wollte Jakob seinen Bruder Esau ein Versöhnungsgeschenk überreichen und bestand hartnäckig darauf, dass dieser es auch als solches annimmt, wodurch er zum Ausdruck bringt, dass er seine Schuld am Konflikt eingesteht und es mit der Versöhnung echt meint (Gen 33, 8-11).

Ein Versöhnungsprozess kann auch in eine übertriebene, ja extreme Nähe hineinschleichen, was wiederum zu neuen Konflikten hinführen kann, wodurch für selbständige Persönlichkeiten ihr eigenständiger Lebensraum zu sehr eingeengt wird. So ließ Jakob seinen Bruder Esau weder mitgehen, noch wollte er von ihm Hilfe annehmen (Gen 33, 12-15). Er beharrte weiterhin auf eigenem Lebensweg, wollte sich auch nicht im Lebensbereich seines Bruders Esau niederlassen, sondern verschaffte sich als selbständiger und verantwortungsbewusster Ehemann, Vater und „Hausherr" ehrenhafterweise selbst seinen eigenen Lebensraum ${ }^{83}$. Diesen wollte er weder geschenkt bekommen noch durch Betrug ergattern. Diesmal ist er ohne weiteres bereit, seine Ersparnisse zu opfern und den neuen „Grundboden“ ehrenhaft zu kaufen, damit der Neubeginn, sein Haus nicht auf Sand, sondern auf sicheren Felsen gebaut werden kann. Da konsolidierte er sämtliche Beziehungen - zu Natur und Tieren, zu sich selbst, seinen Familienmitgliedern und Mitmenschen sowie zu Gott. Am Ende des Versöhnungsprozesses Jakobs, der zugleich ein Prozess zu integrierter Persönlichkeit ist, lesen wir, wie er „wohlbehalten“ zum Neubeginn kommt, wodurch die Bibel zum Ausdruck bringt, wie heilend Integrationsprozesse des eigenen Schattens sein können.

82 „Die Mägde und ihre Kinder kamen näher und warfen sich nieder. Dann kamen auch Lea und ihre Kinder und warfen sich nieder, und zuletzt kamen Josef und Rahel und warfen sich nieder". Gen 33, 6-7.

${ }^{83}$ Jakob gelangte, als er aus Paddan-Aram kam, wohlbehalten bis Sichem in Kanaan und schlug vor der Stadt sein Lager auf. Das Grundstück, auf dem er sein Zelt aufspannte, erwarb er von den Söhnen Hamors, des Vaters von Sichem, für hundert Kesita. Dort errichtete er einen Altar und nannte ihn: Gott, der Herr Israels. Gen 33,19-20. 


\section{Vierte Generation: Josef aus Ägypten}

„Jakob hatte zwölf Söhne“. Ähnlich wie Sara, die Frau Abrahams, und Rebekka, die Frau Isaaks, war auch Rahel, die ,geliebte“ Frau Jakobs, eine lange Zeit unfruchtbar. So war Josef, der Sohn Rahels, in einem gewissen Sinne sein „erstgeborener“ Sohn. Ihn liebte Jakob am meisten ${ }^{84}$, was erwartungsgemäß zu Geschwisterkonflikten führte ${ }^{85}$. Josef wusste die Zuneigungen seines Vaters auszunutzen, was die Beziehungskluft zu seinen Brüdern noch vergrößerte ${ }^{86}$.

Mit dem Heranwachsen stieg Josef die Zuneigung von Seiten seines Vaters bereits ins Unterbewusstsein, was durch seine Träume immer wieder ans Tageslicht gebracht wurde und was seine Brüder zunehmend schwer ertragen konnten (Gen 37, 5-11). So waren sie entschlossen, sich an ihm zu rächen. Sie wollten ihn bei der ersten Gelegenheit umbringen, taten es aber auf Anraten ihres ältesten Bruders Ruben nicht. Sie warfen ihn zuerst in eine Zisterne, die allerdings leer war. Als bei ihnen Leute vorbeikamen, die nach Ägypten unterwegs waren, verkauften sie ihn an sie (Gen 37, 12-36). So kam Josef nach Ägypten.

Josef begann seine erfolgreiche Laufbahn in Ägypten als Sklave. Bereits als Sklave wurde er erfolgreich und machte sich bemerkbar (Gen 39, 2a). Nach einem ihm angedichteten Geschlechtsverkehr mit der Frau seines Hausherren wurde er zwar ins Gefängnis gebracht (Gen 39, 19-20), aber auch dort gelang ihm alles, was er unternahm. Vor allem wurde er durch seine Traumdeutungen bekannt. Infolgedessen kam er bald aus dem Gefängnis heraus und durfte - nach seiner guten Deutung des Traumes Pharaos über die anbrechenden fruchtbaren und bevorstehenden mageren Jahre - sogar bei ihm bleiben (Gen 40, 8-41). So bekam Josef in Ägypten eine verantwortungsvolle Machtposition ${ }^{87}$.

Auf Grund seiner guten Bevorratung konnte die Krise überbrückt werden. Da gleichzeitig auch in seiner alten Heimat eine Hungersnot herrschte, kamen

84 „Israel liebte Josef unter allen Söhnen am meisten, weil er ihm noch in hohem Alter geboren worden war. Er ließ ihm einen Ärmelrock machen“. Gen 37, 3.

85 „Als seine Brüder sahen, dass ihr Vater ihn mehr liebte als alle seine Brüder, hassten sie ihn und konnten mit ihm kein gutes Wort reden“. Gen 37, 4.

${ }^{86}$ „Das ist die Geschlechterfolge nach Jakob: Als Josef siebzehn Jahre alt war, weidete er mit seinen Brüdern, den Söhnen Bilhas und Silpas, der Frauen seines Vaters, die Schafe und Ziegen. Josef hinterbrachte ihrem Vater, was die Brüder Böses taten“. Gen 37, 2.

${ }^{87}$ „Du sollst über meinem Hause stehen, und deinem Wort soll sich mein ganzes Volk beugen. Nur um den Thron will ich höher sein als du. Der Pharao sagte weiter zu Josef: Hiermit stelle ich dich über ganz Ägypten“. Gen 41, 40-41. 
seine Brüder nach Ägypten, um Getreide zu kaufen. Wenn er sich auch nicht gleich zu erkennen gab, so behandelte er sie doch als seine Brüder und sorgte für sie (Gen 42). Die Tatsache, dass er seine Brüder auf die Probe stellte, verhalf ihnen in erster Linie zur eigenen Besinnung.

Als sich Josef beim nächsten Besuch zu erkennen gab und vor allem, als anschließend die ganze Familie Jakobs zu Josef nach Ägypten zog, begann für den gesamten Stamm die bis Abraham zurückreichende Erinnerungsarbeit. Jakob hatte seinen Lieblingssohn bereits ,,verloren“ und doch rettete dieser ihm das Leben.

Die Parallele zur Geschichte über „Abrahams Opfer“, wo Abraham seinen Sohn Isaak (Jakobs Vater) beinahe verloren hätte, um ihn wieder zu ,gewinnen“, liegt da unheimlich nahe. Die krankhaft habsüchtige Vaterbeziehung zum Erstgeborenen ist nicht nur für Abraham, sondern genauso für Isaak und Jakob charakteristisch. Dementsprechend sind auch die Geschwisterkonflikte zwischen Esau und Jakob einerseits sowie Josef und seinen Brüdern andererseits zumindest „verwandt“. Die zum Schluss erbrachte Verzeihungskraft Josefs, als er die Versöhnungsgeste seiner Brüder annahm, ist also tiefgreifend und mehrschichtig ${ }^{88}$. Es ist eine Verzeihung seinen Brüdern, zugleich aber auch seinem Vater gegenüber. Denn, eine andere, weniger habituelle „Vater-Sohn-Beziehung“ hätte auch eine andere, weniger konfliktreiche Familienkonstellation erbracht.

Seine Verzeihungstat reichte aber in einem gewissen Sinne bis Abraham zurück, denn mit dessen „Untreuetat“ wurden ja die mehrere Generationen hindurch andauernden konfliktgeladenen Familienverhältnisse ausgelöst. Erstaunlicherweise wurde die konfliktbereinigende Verzeihungstat Josefs im gleichen Land, d. h. im gleichen „Lebensraum“ vollbracht, wo die „ErbUntat" Abrahams begangen worden war, nämlich in Ägypten.

\section{Schlusswort}

Wie zu biblischen Zeiten ist auch heute ein vollkommenes und konfliktfreies Leben nicht realisierbar. Dramatische Schilderungen der Beziehungen

88 „Ihr habt Böses im Sinn gehabt, Gott aber hatte dabei Gutes im Sinn, um zu erreichen, was heute geschieht: viel Volk am Leben zu erhalten. Nun also fürchtet euch nicht! Ich will für euch und eure Kinder sorgen. So tröstete er sie und redete ihnen freundlich zu“. Gen 50, $20-21$. 
biblischer Familien lehren uns, in Erziehungs- und Kommunikationsprozessen Lebenskonflikte nicht zu ignorieren und diesen nicht auszuweichen, sondern vielmehr als Herausforderungen für gegenwärtige Lernprozesse anzunehmen, die uns dadurch zu innerlicher Transformation bewegen und zur gemeinschaftstiftenden Integration verhelfen können ${ }^{89}$. Wenn uns die Bibel lehrt, Konflikte zu transzendieren und unseren Blick mit der theologischen Dimension der Erlösungsgeschichte zu erweitern, so hindert sie uns nicht daran, manche unheimlich gezielt formulierte praktische Herausforderungen und Orientierungslinien für die Erziehung heute vorzufinden und abzulesen.

Dementsprechend lassen sich Konflikte nur dort (in gleichen oder zumindest ähnlichen Lebensumständen) bereinigen, wo sie entstanden sind, und zwar selbst in Fällen, wenn sich diese mehrere Generationen hindurch ziehen. Gleichzeitig bringen uns die Bibelgeschichten nahe, dass es bei schwerwiegenden Konflikten nicht immer möglich ist, diese innerhalb einer Generation zu bewältigen. Manchmal kommt es zum sogenannten kompulsiven Wiederholungsdruck, wo die kommende Generation imstande ist, Konflikte höchstens $\mathrm{zu}$ ertragen und nur teilweise $\mathrm{zu}$ verarbeiten. Dass sich dabei sowohl Einzelpersonen als auch gesamte Familien im Wandlungsprozessen befinden, die sich in Richtung Integration und Offenheit bewegen, ist ohne Zweifel. Wenn solche „Verdammung“ der Bibel nach bis zu vier Generationen dauert, geht der Segen bzw. die lebensstiftende Kraft nie verloren. Im Gegenteil, „Verdammung“, die uns verwundbar und damit offen und sensibel macht, wird selbst zum Segen ${ }^{90}$. Dies trifft vor allem dann zu, wenn - aus Liebe zu ihren Kindern - die Eltern befähigt werden, eigene Probleme zu lösen und für eigenes Wohl zu sorgen: „Das meiste, das die gegenwärtigen Eltern für ihre Kinder tun können ist dafür zu sorgen, dass sie selber vor allem auf der emotionalen Ebene ein zufriedenes und gelungenes Leben führen" $"$.911.

Auf der einen Seite könnte der Eindruck entstehen, solche biblische Geschichten belasten den Menschen, denn niemand ist fehlerfrei und auch nicht von schmerzhaften Fehlentscheidungen dispensiert. In der Tat ist aber gerade das Gegenteil der Fall. Bibeltexte beweisen, dass der Mensch nie so tief fallen kann, dass er - wenn er hofft, an eine Lösung glaubt und

\footnotetext{
${ }^{89}$ Vgl. R. D. Bell, Biblical Models of Handling Conflict, Vancouver 2001, S. 13-15.

${ }^{90} \mathrm{Vgl}$. R. W. Firestone, J. Catlett, The Ethics of Interpersonal Relationships, London 2009, S. 33.

${ }^{91}$ R. W. Firestone, J. Catlett, The Ethics of Interpersonal Relationships, London 2009, S. 65.
} 
offen kommuniziert - nicht imstande wäre, aus noch so schmerzhafter Lebenssituation als verwandelter Mensch herauszukommen. Die extrem dramatischen Schilderungen der Lebensfragen sind also dazu da, um Hoffnung zu geben, dass es im Leben nie zu spät ist, etwas ändern zu wollen. In der Bibel bleiben nur jene Menschen auf der Strecke, die sich in sich einsperren. Alle, die offen kommunizieren, finden einen Ausweg.

\section{Bibliography}

Bell R. D., Biblical Models of Handling Conflict, Vancouver 2001, Regent College Publishing.

Cloud H., Townsend J., How People Grow: What the Bible Reveals about Personal Growth, Grand Rapids - Michigan 2001, Zondervan.

Cohen N. J., Self, Struggle \& Change: Family Conflict Stories in Genesis and Their Healing Insights for Our Lives, Woodstock 1995, Jewish Lights Publishing.

Cohen N. J., Voices from Genesis: Guiding Us Through the Stages of Life. Woodstock-Vermont 1998, Jewish Lights Publishing.

Corey M. A., Job, Jonah, and the Unconscious: A Psychological Interpretation of Evil and Spiritual Growth in the Old Testament, Lanham - New York - London 1995, University Press of America.

Creach J. F. D., Violence in Scripture, Louisville - Kentucky 2013, Westminster John Knox Press.

Firestone R. W., Catlett J., The Ethics of Interpersonal Relationships, London 2009, Karnac.

Forgas J. P., Affective Influences on Communication and Attribution in Relationships, in: V. Manusov, J. H. Harvey (Hrsg.), Attribution, Communication Behavior, and Close Relationships, Cambridge - New York - Melbourne - Madrid - Cape Town - Singapore - Sao Paulo - Delhi - Tokyo - Mexico City 2001, Cambridge University Press, S. 3-20.

Friedman R. E., Commentary on the Torah: With a new English Translation, San Francisco 2001, Harper Collins Publishers.

Gerjolj S., Živeti, delati, ljubiti: Pedagoška in psihološka interpretacija svetopisemskih pripovedi, Celje 2009, Celjska Mohorjeva družba.

Hartley E. J., New International Biblical Commentary: Genesis, Peabody - Massachusetts - United Kingdom 2000, Hendrickson Publishers, Paternoster Press.

Höfer A., Gottes Wege mit den Menschen: Ein gestaltpädagogisches Bibelwerkbuch, München 1993, Don Bosco Verlag.

Höfer A., Spuren Gottes in meinem Leben, München 2003, Don Bosco Verlag.

Kille A. L., Jacob: A Study in Individuation, in: D. L. Miller (Hrsg.), Jung and the Interpretation of the Bible, New York 1995, Continuum, S. 40-54.

Loder J. E., The Logic of the Spirit: Human Development in Theological Perspective, San Francisco 1998, Jossey-Bass Publishers. 
Powell L., Self W. R., Holy Murder: Abraham, Isaac, and the Rhetoric of Sacrifice, Lanham - Boulder - New York - Toronto - Plymouth 2007, University Press of America.

Pravder Mirkin M., The Woman Who Danced By The Sea: Finding Ourselves in the Stories of our Biblical Foremothers, New York 2005, Monkfish Book Publishing Company.

Rollins W. G., Soul and Psyche: The Bible in Psychological Perspective, Minneapolis 1999, Augsburg Fortress.

Schirmer E., Müttergeschichten: Frauen aus dem Alten Testament erzählen von ihrem Leben, Offenbach 1989, Jünger Medien \& Burckhardthaus-Laetere.

Trible P., Ominous Beginnings for a Promise of Blessing, in: P. Trible, L. M. Russell (Hrsg.), Hagar, Sarah, and Their Children, Louisville - Kentucky 2006, Westminster John Knox Press, S. 33-69.

Walton H. J., The NIV Application Commentary: From biblical text ... to contemporary life, Grand Rapids - Michigan 2001, Zondervan. 